\begin{tabular}{l}
\hline Jurnal Kesehatan Masyarakat \\
http://journal.unnes.ac.id/nju/index.php/kemas
\end{tabular}

\title{
GENDER-BASED HEALTH DISORDERS IN END-STAGE RENAL DISEASE PATIENTS IN THE HOSPITAL ELMARJ CITY LIBYA
}

\author{
Emad Mousay Mohammed Amkhatirah ${ }^{1 凶}$, Ari Yuniastuti $^{2}$ \\ 1Elmarj, Libya \\ ${ }_{2}$ Universitas Negeri Semarang
}

\section{Info Artikel \\ Article History: \\ Submitted April 2017 \\ Accepted July 2017 \\ Published July 2017 \\ Keywords: \\ End-Stage Kidney Disease \\ (ESRD); Hypertension; \\ Diabetes Mellitus; Urinary \\ Tract Infection; Urinary \\ Tract Stone; Obesity \\ DOI \\ http://dx.doi.org/10.15294/ \\ kemas.v13i1.9413}

\begin{abstract}
End-stage renal disease is the last stage of chronic kidney disease, hence a dangerous disease and poses many problems for the patients. The aim of this study was to analyze the risk factors for end-stage renal disease in Elmarj, Libya. Data was collected by distributing questionnaires to the respondents. The questionnaire was a set of written questions used to obtain information from respondents, be it their personal statements or things that they knew. The questionnaires were distributed to the respondents in person; the researchers distributed questionnaires to the respondents. The aim of this research is to determine the risk factors associated with ESRD (hypertension, DM, urinary tract infection, urinary tract stone, and obesity) among patients with ESRD the hospital Elmarj City Libya. This can be seen as the descriptive risk factors.
\end{abstract}

\section{Introduction}

End-stage kidney disease is the last stage of chronic kidney disease. This happens when the kidneys can no longer support the body's needs. The kidneys remove waste and excess water from the body. End-stage kidney disease is also called end-stage renal disease (ESRD). There are some causes of ESRD occurs when the kidneys are no longer able to work at a level needed for day-to-day life (Serra, 2008; Supriyadi, 2011).

The most common causes of ESRD in the U.S. are diabetes and high blood pressure. The common symptoms may include: generalized fatigue, itching and dry skin, headache, unexplained weight loss, loss of appetite, nausea. Other symptoms may include: abnormally dark or light skin, nail changes, bone pain, drowsiness and confusion, problems in concentrating or thinking, numbness in the extremities, muscle twitching or cramps, breath odor, easy bruising, nosebleeds, blood in the stool, excessive thirst, frequent hiccups, sexual dysfunction, amenorrhea, sleep disturbances, edema in the feet and hands, vomiting, often in the morning (Schlondorff, 2008).

Diabetes is the leading cause of chronic renal disease, it is responsible for $33 \%$ of adults with chronic renal (Mariusz, 2013). Nevertheless, $20 \%$ to $40 \%$ of diabetics will develop diabetic nephropathy during the end stage of their disease. Therefore, as the number 
of diabetes cases increase, the incidence of CKD is expected to rise. The initial presentation of diabetic kidney disease is microalbuminuria followed by increasing severity of proteinuria as the glomerular filtration membrane is damaged (Ernesto, 2007). Hypertension represents a powerful risk factor for chronic renal disease and is almost always found in patients with renal failure. Sodium retention and activation of the renin-angiotensin system have been considered as the most effective mechanisms implicated in the rising of blood pressure in patients with chronic renal disease (Guyton, 2009).

Obesity has been acknowledged as a risk factor for the development of chronic renal disease, independently of hypertension, diabetes, and pre-existing renal disease (Ejerbald, 2006). Obesity often coexists with hypertension, which may cause nephropathy (Mariusz, 2013). Obesity is associated with the early onset of glomerulomegaly, hemodynamic changes of a hyperfiltering kidney, and increased albuminuria, which are reversible with weight loss (Eknoyan, 2011).

Previous research conducted by Traina (2015), was a descriptive study conducted retrospectively by reviewing the files of all patients attending haemodialysis unit at Misurata Central Hospital during 2005. The total number of ESRD patients undergoing haemodialysis from January 2005 to December in the same year was 70 patients. Their mean age was $36.4 \pm 17.7$ years with a median 37.7 (range 3-69). Male constituted $55.7 \%$ of patients while female patients were $44.3 \%$ with male to female ratio of $1.25: 1$. The estimated prevalence of ESRD was $34.71 \%$ patients per 100.000 populations. The most common causes of ESRD were diabetes mellitus in $28.5 \%$ and hypertension in $17.1 \%$ followed in order by undetermined causes in $15.7 \%$, glomerulonephritis in $14.3 \%$, chronic pyelonephritis in $8.6 \%$, nephrotoxic drugs in $5.7 \%$. Other less common causes such as obstructive uropathy, polycystic kidney disease, gout and IgA nephropathy also were reported in our study. The most common cause of ESRD were found to be Diabetes Mellitus (DM) and HTN, affecting the younger population.

Eknoyan (2011), stated that the incidence and prevalence of ESRD in the United States are expected to increase by
$44 \%$ and $85 \%$, respectively, from 2000 to 2015. Reducing the incidence of ESRD is widely recognized. However, few longitudinal cohort studies focusing on identifying risk factors for ESRD have been conducted.The goal of this study was to evaluate the prognostic value of several potential novel risk factors for ESRD after considering established risk factors. Simultaneous assessment is important because some of the proposed novel risk factors (eg, high serum uric acid level or low hemoglobin level) may be highly correlated with established risk factors. Identification of novel risk factors that predict ESRD independent of traditional risk factors may help improve risk stratification and facilitate more focused preventive efforts among higher-risk subgroups. It may also uncover new potential therapeutic targets to combat the rising tide of ESRD.

Therefore, this research would analyze risk factors associated end-stage renal disease in Elmarj, Libya.

\section{Method}

The population in this research was all patients in Elmarj hospital, Lybia. The eligible population was 83 patients suffering from ERSD in Elmarj hospital, Libya.

Independent variables are risk factors that consist of hypertension (is the condition of patients that have high blood pressure), diabetes mellitus (is the condition of patients that have high serum glocuse), urinary tract infection (is the condition of patients that suffer from urinary tract infection), urinary tract stone (is the condition of patients that suffer from urinary tract stone), and obesity (is the condition of patients that suffer from obesity). Dependent variable is ESRD is the condition of patients that suffer from renal disease.

The following were procedures used in this study to find the respondents. The study was done in Elmarj hospital, Libya, questionnaire was developed according to the purpose of the study, the questionnaire was reviewed and improved, and lastly distributing the questionnaires.

Data was collected by distributing questionnaires to the respondents. All data was analyzed using the statistical package for social science (SPSS program). This study is a retrospective descriptive study done by 
reviewing the medical records of all patients attending Haemodialysis unit at Elmarj hospital, Libya during the year of 2016.

\section{Results and Discussion}

From the table above, it could be seen that there was more male than the female participants. From a total of 83 participants, $80,7 \%$ were male (67) while $19,3 \%$ were females (16). As shown in the Table 4.1 10,8\% participants were $<30$ years old, $48,2 \%$ were $<50$ years old and $41 \%$ were $30-50$ years old.

In this section the risk factors associated with ESRD, such as hypertension, diabetes mellitus, urinary tract infection, urinary tract stone and obesity will be discussed.

From the table 1 it can be seen that majority of the respondents were male without hypertension $(63.9 \%)$ and female without hypertension (18.1\%). Hypertensive female were only $1.2 \%$ while male were $16.9 \%$. Therefore, hypertension tend to dominate among male because their behavior or the habit of eating more than female.

Table 1. Gender and Hypertension Respondents

\begin{tabular}{lll}
\hline & \multicolumn{2}{l}{ Gender } \\
\cline { 2 - 3 } & Female & Male \\
\hline Hypertention & & \\
No & 15 & 53 \\
Yes & 1 & 15 \\
\hline Diabetes Mellitus & & \\
No & 5 & 30 \\
Yes & 11 & 37 \\
\hline Urinary Tract Infection & \\
No & 4 & 58 \\
Yes & 12 & 9 \\
\hline Urinary Tract Stones & \\
No & 8 & 35 \\
Yes & 8 & 32 \\
\hline Obesity & & \\
No & 8 & 31 \\
Yes & 8 & 36 \\
\hline
\end{tabular}

Source : Primary Data

From the table 1 it can be seen that majority of the respondents were male with diabetes mellitus (44.6\%) and female with DM
(13.3\%). Total respondent suffering from DM were 48 persons $(57.8 \%)$ and the remaing 35 respondents did not suffer from DM. More male suffer from diabetes mellitus because Libya male ate more carbohydrate in their food, hence predisposes them to diabetes mellitus. This is not the same with females because they could maintain their lifestyle to avoid being overweight and they tend to eat less carbohydrates in their food.

From the table 1 it can be seen that the majority of respondents were female with no urinary tract infection $(50.6 \%)$ and male with no urinary tract infection (8.4\%). Total respondent suffering from urinary tract infection wre 21 persons (38.6\%) and remaing 62 respondents did not suffer from urinary tract infection. Therefore more female suffered from urinary tract infection than male because they are more prone to be infected by bacteria.

From the table 1 it can be seen that majority of the respondents were male with no urinary tract stones $(42.2 \%)$ and female with urinary tract stones $(9.6 \%)$. Total respondent suffering from urinary tract stones were 43 persons $(51.8 \%)$ and the remaining 40 respondents did not suffer from urinary tract stones. More male suffer from urinary tract stones because of the lifestyle and the influence of hormones and degree of physical activity.

From the table 1 it can be seen that majority of the respondents were male with obesity (53\%) and female with obesity (9.6\%). Total number of respondents suffering from obesity were 44 persons $(53 \%)$ and the remaining 39 respondents were not obese (47\%). The reason why more male were obese was because of their lifestyle and the lack of physical activity, while females tend to have more physical activity such as exercises, brisk walking, cycling, as well as activities that strengthen bones and muscles such as jumping rope, gymnastics or sit ups.

From the questionnaires' answers, it could be seen that the majority of respondents were male. From a total of 83 participants, $80,7 \%$ were male (67), while $19,3 \%$ were females (16). The majority of participants were in senior high school $(54,2 \%)$ and $45,8 \%$ had bachelor degree. The majority of participants were $<50$ years old $(48,2 \%)$ while the remaining were $30-50$ years 
old $(41 \%)$ and $<30$ years old $(10,8 \%)$.

Renal disease is a pathological process with diverse etiology, resulting in a progressive decline in renal function, and generally ends with kidney failure. Furthermore, renal failure is a clinical condition characterized by the irreversible decline in kidney function, to a degree that requires renal replacement therapy, in the form of dialysis or kidney transplantation (Schieffer, 2004). According Shen (2010), renal disease or chronic renal failure is a progressive renal damage which is fatal and characterized by uremia (urea and nitrogenous wastes other circulating in the blood and complications if not done dialysis or a kidney transplant (Hussain, 2015). Chronic renal failure (CRF) or end-stage renal disease is a disorder of renal function which is progressive and irreversible where the ability of the body to excrete fails to maintain the normal level of metabolic by-products and maintain fluid and electrolyte balance, causing uremia (retention of urea and wastes other nitrogen in the blood).

According to Wesson (2005), chronic kidney failure or end stage renal disease (ESRD) is a progressive renal function impairment and irreversible failure where the body's ability to metabolism and maintain fluid and electrolyte balance, causing uremia (retention of urea and other nitrogen wastes in the blood) (Pranav, 2013).

When discussing the pathophysiology of ESRD, renal structural and physiological characteristics, as well as the principles of renal tissue injury and repair should be taken into consideration. Firstly, the rate of renal blood flow of approximately $400 \mathrm{ml} / 100 \mathrm{~g}$ of tissue per minute, which is much greater than that observed in other well perfused vascular beds such as heart, liver and brain. As a consequence, renal tissue might be exposed to a significant quantity of any potentially harmful circulating agents or substances (Ibrahim, 2012).

Secondly, glomerular filtration is dependent on a rather high intra and transglomerular pressure, rendering the glomerular capillaries vulnerable to hemodynamic injury, in contrast to other capillary beds. In line with this, Brenner and coworkers identified glomerular hypertension and hyperfiltration as major contributors to the progression of chronic renal disease.

Thirdly, glomerular filtration membrane has negatively charged molecules which serve as a barrier retarding anionic macromolecules (Özkan, 2015). With disruption in this electrostatic barrier, as is the case in many forms of glomerular injury, plasma protein gains access into the glomerular filtrate.

Fourthly, the sequential organization of nephron's microvasculature (glomerular convolute and the peritubular capillary network) and the downstream position of the tubuli with respect to glomeruli, not only maintains the glomerulo-tubular balance but also facilitates the spreading of glomerular injury to tubulointerstitial compartment in disease, exposing tubular epithelial cells to abnormal ultrafiltrate. As peritubular vasculature underlies glomerular circulation, some mediators of glomerular inflammatory reaction may overflow into the peritubular circulation contributing to the interstitial inflammatory reaction frequently recorded in glomerular disease (Özkan, 2015).

Moreover, any decrease in preglomerular or glomerular perfusion leads to decrease in peritubular blood flow, which, depending on the degree of hypoxia, entails tubulointerstitial injury and tissue remodeling. Thus, the concept of the nephron as a functional unit applies not only to renal physiology, but also to the pathophysiology of renal diseases. In the fifth place, the glomerulus itself should also be regarded as a functional unit with each of its individual constituents, i.e. endothothelial, mesangial, visceral and parietal epithelial cells - podocytes, and their extracellular matrix representing an integral part of the normal function (Özkan, 2015). Damage to one will in part affect the other through different mechanisms, direct cell-cell connections (e.g., gap junctions), soluble mediators such as chemokines, cytokines, growth factors, and changes in matrix and basement membrane composition. The main causes of renal injury are based on immunologic reactions (initiated by immune complexes or immune cells), tissue hypoxia and ischaemia, exogenic agents like drugs, endogenous substances like glucose or paraproteins and others, and genetic defects. Irrespective of the underlying 
cause glomerulosclerosis and tubulointerstitial fibrosis are common to ESRD (Almutary, 2013). An overview of the pathophysiology of ESRD should give special consideration to mechanisms of glomerular, tubular and vascular injury (Hussain, 2015).

The management of complications of ESRD, such as anaemia and bone disease, is also discussed. Evidence for the best psychological and social support for patients and what information they need to take an optimal part in the management of their condition has been identified and incorporated. The management of patients with ESRD or patients with acute kidney disease is excluded from this discussion. Patients with clinical features suggestive of a primary renal diagnosis, e.g. glomerulonephritis presenting with nephrotic syndrome, or renal disease secondary to vasculitis presenting with haematuria and proteinuria, should be referred to the renal specialists. Their specific management is not part of this guideline. The management of complications associated with ESRD during pregnancy is a specialised area which is not covered in this discussion. (Schieffer, 2004).

Diabetic nephropathy is a renal complication of diabetes mellitus. Diabetes is the most common cause of ESRD requiring renal replacement therapy. The age-adjusted incidence of all-cause ESRD among male with diabetes is more than 12 times greater than in male without diabetes.

Patients with ESRD are often prescribed medications for comorbid conditions, such as diabetes. All drug dosages should be adjusted for kidney function, where appropriate. Drugs with potentially adverse effects on kidney function or complications of decreased kidney function should be discontinued if possible. Information on whether drugs are contraindicated in ESRD is available in the current British National Formulary (BNF) and summary of product characteristics (SPC) (Mariusz, 2013).

Hypertension is defined as a systolic blood pressure (SBP) of $140 \mathrm{~mm} \mathrm{Hg}$ or more, or a diastolic blood pressure (DBP) of 90 $\mathrm{mm} \mathrm{Hg}$ or more, or taking antihypertensive medication. Hypertension may be primary, which may develop as a result of environmental or genetic causes, or secondary, which has multiple etiologies, including renal, vascular, and endocrine causes. From the result majority respondents were male with no hypertension $(63.9 \%)$ and females with no hypertension

(18.1\%). Hypertensive females were only $1.2 \%$ and male were only $16.9 \%$. This means that hypertention is a determinant of ESRD.

The natural course of hypertension is very slow. Patients with hypertension may not show symptoms for years. This latency period masks the progression of the disease until significant organ damage occurs (Wesson, 2005). Hypertension can cause GGT through a process that resulted in the progressive and irreversible loss of a large number of functional nephron. The decline in the number of nephrons will cause adaptive process, which increased blood flow, the progressive increase in GFR (Glomerular Filtration Rate) and an increase in urine output in the nephron is still remains. This process involves hypertrophy and vasodilation of nephrons and decreased vascular resistance and tubular reabsorption in the surviving nephrons. In the long term, the sclerotic lesions formed from each damaged nephron can lead to the obliteration of the glomeruli, resulting in further decrease of kidney function, creating a vicious circle that can develop slowly and ends as in terminal kidney disease (Guyton, 2009). Almutary (2013) also stated that the effect of hypertension on the kidney depends on hypertension and a history of hypertension. The higher the blood pressure and the longer the duration of hypertension, the more severe complications that arise, especially in the kidneys.

In Libya, based on the result of this study, hypertension occurred more among male than females because of their behavior and the fact that they ate more than female. male also have more risk factors for hypertension including smoking, emotional stress and alcohol consumtion, hence male (male) has higher risk of developing hypertension compared to females (Guyton and Hall, 2007).

Diabetes mellitus is a group of metabolic diseases characterized by hyperglycemia resulting from defects in insulin secretion, insulin action, or both. The chronic hyperglycemia of diabetes is associated with long-term organ damage, dysfunction and 
failure, especially the eyes, kidneys, nerves, heart, and blood vessels. Long-term complications of diabetes include retinopathy with potential loss of vision; nephropathy leading to renal failure; peripheral neuropathy with risk of foot ulcers, amputations, and Charcot joints; and autonomic neuropathy causing gastrointestinal, genitourinary, and cardiovascular symptoms and sexual dysfunction. From the result, majority of the respondents were male with diabetes mellitus $(44.6 \%)$ and female with DM (13.3\%). Total respondent suffering from DM were 48 (57.8\%), the remaining 35 respondents were no DM; hence diabetes mellitus is a determinant of ESRD.

In addition to gender-risk for the patients that suffered from diabetes mellitus and terminal renal failure, age is also a risk factor for damage of diabetes mellitus and terminal renal failure. From the results, it was found that the age most often coincided with diabetes mellitus resulting in terminal kidney failure were the age of 40-55 years. Among the degenerative diseases, diabetes is one of the non-communicable disease predicted to increase in the future. Diabetes is already one of the main threats to human health in the 21st century. The World Health Organizations (WHO) made a forecast that in 2000 the number of people with diabetes aged $>20$ years amounts to 150 million people and 25 years later, in 2025, that number would swell to 300 million people (Serra, 2008).

In Libya, based on the result of this study, male had higher risk of developing Diabetes Mellitus than females because in Libya male eat more carbohydrate in their food, hence increasing the risk for diabetes mellitus, meanwhile females tend to maintain their weight and eat less carbohydrate in their food (Taylor, 2005).

A urinary tract infection (UTI) is an infection in any part of urinary system from the kidneys, ureters, bladder and urethra. Most infections involve the lower urinary tract, the bladder and the urethra. Women have greater risk of developing UTI compared to males. Infection limited to the bladder may be painful and disturbing. However, serious consequences can occur if the infection spreads to the kidneys. The most common cause of infection is Escherichia coli, though other bacteria or fungi may rarely be the cause. Risk factors include female anatomy, sexual intercourse, diabetes, obesity, and family history. The result show that majority of the respondents were male with no urinary tract infection $(50.6 \%)$ and females with no urinary tract infection (8.4\%). Total respondent suffering from urinary tract infection were $32(38.6 \%)$, the remaining 51 respondents had no urinary tract infection. Hence, urinary tract infection is a determinant of ESRD.

In Libya, based on the result of this study, more female suffered from UTI than male. The reason is because the infection begins with the entry of bacteria into the urinary hole and continue to spread upwards towards the kidney. Hence, bacteria have to move against the flow of urine that flows from the kidneys to the ureter, into the bladder and eventually expelled through the urethra (Schlondorff, 2008).

Renal stone is a stone in the kidney (or lower down in the urinary tract); also called a kidney stone. Renal stones are a common cause of hematuria and abdominal pain, flank pain, or pain in the groin. Kidney stones occur in 1 out of 20 people at some time in their life. The development of the stones is related to decreased urine volume or increased excretion of stone-forming components such as calcium, oxalate, urate, cystine, xanthine, and phosphate. The pain caused by kidney stones is usually of sudden onset, very severe and colicky (intermittent), not improved by changes in position, radiating from the back, down the flank, and into the groin. The result showed that the majority of respondents were male with no urinary tract stones $(42.2 \%)$ and female with urinary tract stones $(9.6 \%)$. Total respondent suffering from urinary tract stones were $43(51.8 \%)$, the remaining 40 had no urinary tract stones and this means that urinary tract stone is a determinant of ESRD.

In Libya, more male suffered from urinary tract stones than female. This is because of hormonal influence and physical activity. Male urinary tract is narrower than that in female, causing kidney stones to easily clog the tract, causing the problem. The kidney stones can actually be asymptomatic and may cause complications even when they were successfully expelled (Shen, 2010). 
Obesity is associated with multiple other conditions that are known to cause compromised renal function, including hypertension, diabetes, hyperuricemia, and the metabolic syndrome that can independently have a detrimental effect on renal function (Mariusz, 2013). However, as shown by the evidence above, obesity has been found to be a risk factor for kidney disease and ESRD even after adjustment for these factors. Hence, the pathogenesis of obesity related kidney disease can be sub-classified into direct and indirect effects. Higher BMI was a risk factor for ESRD in multivariable models was adjusted for age, sex, race, education level, smoking status, history of myocardial infarction, serum cholesterol level, urinalysis proteinuria, urinalysis hematuria, and serum creatinine level. The result showed that majority of the respondents were male with obesity $(53 \%)$ and female with obesity $(9.6 \%)$. Total respondent suffering from obesity were $44(53 \%)$, the remaining 39 were not obese $(47 \%)$ and this means that obesity is a determinant of ESRD.

In Libya, based on the result of this study, male tend to be more obese than female because the lifestyle, behaviour, the culture. Such examples would be male prefer to eat out, eat more meat, and also like to eat fast food for lunch because their busy schedule (Eknoyan, 2011).

\section{Conclusion}

Based on the result and the analysis, the determinants of ESRD are hypertension, DM, urinary tract infection, urinary tract stone, and obesity. More male suffer from hypertension compared to female because of the lifestyle in Libya. Diabetes mellitus, urinary tract stones, obesity were higher among male than female because the culture, lifestyle, and behavior in Libya. However, more female suffered from urinary tract infection than male because their urethra is more easily infected by the bacteria.

\section{References}

Almutary, H., Ann Bonner and Clint Douglas. 2013. Chronic Kidney Disease in Saudi Arabia: A Nursing Perspective. Middle East Journal Of Nursing, 7 (6).

Ejerblad E, Fored CM, Lindblad P. 2006. Obesity and Risk for Chronic Renal Failure. $J$ Am SocNephrol, 17 : 1695-702.

Eknoyan G. 2011. Obesity and Chronic Kidney
Disease. Nefrologia, 31(4) : 397-403.

Ernesto L. Schiffrin, Mark L. Lipman, Johannes F.E. Mann. 2007. Cardiovascular Involvement in General Medical Conditions; Chronic Kidney Disease: Effects on the Cardiovascular System. Circulation, $116:$ 85-97.

Guyton AC, Coleman TG, Wilcox CS. 2009. Quantitative Analysis of the Pathophysiology of Hypertension. $J \mathrm{Am}$ SocNephrol, 10 : 2248- 2249.

Hussain Gadelkarim Ahmed, et al. 2015. Association of Genetic and Biochemical Markers with GFR among Renal Failure Patients: Applying Serum Creatinine and Cystatin $\mathrm{C}$ measures. Scholars Journal of Applied Medical Sciences (SJAMS) ISSN 2320-6691 (Online) Sch. J. App. Med. Sci., 3 (6D) : 2431-2436.

Ibrahim Abdelmajeed Ginawi, Hussain Gadelkarim Ahmed, Awdah M. Al-hazimi. 2012. Assessment of Risk Factors for Chronic Kidney Disease in Saudi Arabia. International Journal of Science and Research (IJSR) ISSN (Online): 2319-7064

Mariusz Stępień, Anna Stępień, Rafał Nikodem Wlazel. 2013. Obesity Indices and Adipokines in Non-diabetic Obese Patients with Early Stages of Chronic Kidney Disease. Med SciMonit, 19 : 1063-1072.

Özkan, G, Şükrü Ulusoy. 2015. A Case of Renal Failure Developing in Association with African Mango Consumption. Int J Clin Exp Med, 8 (4) : 6374-6378

Pranav Vashisth, Vinay Jain, Love Singh Chokotia, Rajkumar Sironiya, Harsha Matoli, Megha Jain. 2013. An Update On Herb Induced Chronic Kidney Diseases. International Journal of Research and Development in Pharmacy and Life Sciences, 2 (3) : 428-431

Schlondorff, D. O. 2008. Overview of factors contributing to the pathophysiology of progressive renal disease. Kidney International, 74 (7) : 860-866

Schieffer, B., et al 2004. Comparative Effects of AT1-Antagonism and Angiotensinconverting Enzyme Inhibition on Markers of Inflammation and Platelet Aggregation in Patients with Coronary Artery Disease. $J \mathrm{Am}$ Coll Cardiol, 44 (2) : 362-368.

Serra, A., et al. 2008. Renal Injury in the Extremely Obese Patients with Normal Renal Function, 947-955

ShenW.,etal.2010.Obesity-relatedGlomerulopathy: Body Mass Index and Proteinuria. Clin J Am Soc Nephrol, 5 (8) : 1401-1409.

Supriyadi, Wagiyo, Sekar Ratih Widowati. 2011. Tingkat Kualitas Hidup Pasien Gagal 
Emad M.M.A, \& Ari Yuniastuti / Gender-Based Health Disorders In End-Stage Renal Disease Patients

Ginjal Kronik Terapi Hemodialisis. Jurnal Kesehatan Masyarakat (KEMAS), 6 (2)

Taylor E., et al. 2005. Obesity, Weight Gain, and the Risk of Kidney Stones. JAMA, 293(4) : 455-462.

Traina, Mohamed, Masoud Ghazit, and Farij Traina.
2015. End-Stage Renal Disease In Misurata. MMSJ, 1 (2)

Wesson D., et al. 2005. Massive Obesity and Nephrotic Proteinuria with a Normal Renal Biopsy. Nephron, 40 (2) : 235-237. 